\title{
Status of Infectious Urolithiasis and Multi-resistant Bacteria Extended Spectrum $\beta$-lactamase in Algeria
}

\section{Berrahal M*}

Department of Medical - Microbiology, University 01 Algeria, Kouba, Algeria

*Corresponding Author: Berrahal M, Department of Medical - Microbiology,

University 01 Algeria, Kouba, Algeria.

DOI: $10.31080 /$ ASMS.2022.06.1200
Received: January 07, 2022

Published: February 16, 2022

(C) All rights are reserved by Berrahal $\mathbf{M}$.

\begin{abstract}
Introduction: We wanted to take stock of the new microbio-epidemiological trend of mixed urolithiasis infected or colonized by multi-resistant germs including multi-resistant enterobacteria bacteria extended spectrum $\beta$-lactamase and others.

Materials and Methods: Assess the impact of the microbiological study of the microbiological study (phenotypic and molecular) of pyelic urine and/or stone compared to standard urineculture (ECBU).

Results: 114 (03\%) had an infected (or colonized) stone. Preoperative ECBU was $90 \%$ negative compared at the culture of urine pyelique and stone with 22\% Proteus mirabilis, 15\% Staphylococcus aureus, 11\% K. pneumoniae and others emerging species. Antibiotic resistance detected in the stone and pyelic urine analysis: $60 \%$ of $E$. coli and $40 \%$ of $K$. pneumoniae resistant to Cefazolin (CIIG) with $60 \%$ resistance to ciprofloxacin in E. coli and $20 \%$ in K. pneumoniae. we had five extended spectrum $\beta$-lactamase strain: tow Enterobacter cloacae complex (ECC) and three K. pneumoniae.

Conclusion: Mixed infectious urolithiasis can be a real public health problem because, pathogenic germs sometimes alarmist due to their resistance to antibiotics and their etiopathogens or virulences and can be life-threatening for patients with additional costs, especially hospital and social care.
\end{abstract}

Keywords: Microbio-epidemiological; Extended Spectrum Beta-lactamase; Stone Culture; Pyelic Urine; Antibiotic Resistance; Vital Prognosis

\section{Introduction}

The urolithiasis takes the tangent in our country with $40 \%$ of anatomical anomaly obstructive and considering the operational increase in the gestures [1]. The which place of the urinome, virulome or resistome microorganism implicate in the etiopathogenic of infectious stones diseases and renal failure.

Goal: To assess the impact of the microbiological study of pyelic urine and/or urinary calculus versus ECBU in the detection of multi-resistant bacteria extended spectrum.

$\beta$-lactamase in infectious urolithiasis.

\section{Materials and Methods}

The study was carried out at Mohamed Seghir Nekkache Hos- pital and involved bacterial strains from the urine of 114 patients with infection-colonization of the urinary calculus. We performed 114 preoperative ECBUs, 54\% cytobacteriological examinations of pyelic urine and $65 \%$ bacteriological examinations of urinary calculi (fragments). After enrichment and cultivation (according to Stamey), the identification of positive cultures was made by conventional and automated method followed by antibiograms according to CLSI 2020. In addition, CMI E-Test EBLSE for enterobacteriaceae resistant to Third-generation cephalosporin-resistant enterobacteriaceae, with study of extended spectrum $\beta$-lactamase (EBLSE) genes by PCR and sequencing. MLST genotyping of extended spectrum K. pneumoniae $\beta$-lactamase was found in calculus culture (Annex). 
Molecular study of antibiotic resistance in urinary stone isolate.

Table : Primers used in PCR and molecular study sequencing.

\begin{tabular}{|c|c|c|c|}
\hline Gènes & Amorces & Séquences & $\begin{array}{l}\text { Taille des amplicon } \\
\text { (Bp) }\end{array}$ \\
\hline bla $_{T E M}$ & TEM-F TEM-R & $\begin{array}{l}\text { TTACCAATGCTTAATCA ATAAAATTC- } \\
\text { TTGAAGAC }\end{array}$ & 867 \\
\hline$b l a_{S H V}$ & SHV-F SHV-R & $\begin{array}{l}\text { TGGTTATGCGTTATATTCGCC GCT- } \\
\text { TAGCGTTGCCAGTGCT }\end{array}$ & 1750 \\
\hline$b l a_{P E R}$ & PER F PER R & $\begin{array}{l}\text { GTAGTATCAGCCCAATCCCC CCAA- } \\
\text { TAAAGGCCGTCCATCA }\end{array}$ & 738 \\
\hline$b l a_{\mathrm{VEB}}$ & VEB F VEB R & $\begin{array}{l}\text { GGAACAACTTTGACGATTGA CCC- } \\
\text { TGTTTTATGAGCAACAA }\end{array}$ & 374 \\
\hline bla $_{G E S}$ & GES F GES R & $\begin{array}{l}\text { GAAAAAGCAGCTCAGATCG CAA- } \\
\text { CAACCCAATCTTTAGGA }\end{array}$ & 597 \\
\hline bla $_{\text {CTX-M-13 }}$ & M15-F M15-R & $\begin{array}{l}\text { GGTTAAAAAATCACTGCGTC TTG- } \\
\text { GTGACGATTTTAGCCGC }\end{array}$ & 863 \\
\hline$b^{b l a}{ }_{C T X-M-9}$ & M9-F M9-R & $\begin{array}{l}\text { ATGGTGACAAAGAGAGTGC CCCTTCG- } \\
\text { GCGATGATTCTC }\end{array}$ & 870 \\
\hline $\operatorname{acc}\left(3^{\prime}\right)_{1 b}$ & $\begin{array}{l}\text { acc- } 1 b \\
\text { acc- } 1 b\end{array}$ & $\begin{array}{l}\text { CACCTCCAGCGACTTGTTAC GTTAGC- } \\
\text { CAGCATCACGATCC }\end{array}$ & 346 \\
\hline$q n r$ & qnrB F qnrB F & $\begin{array}{l}\text { TGGCGAAAAAATTGAACAGAA GAG- } \\
\text { CAACGATCGCCTGGTAG }\end{array}$ & 494 \\
\hline
\end{tabular}

The protocol used, the primers and the PCR conditions were carried out according to Diancourt et al. (2018).

Primers for PCR amplification

RpoB : -FVic3: GGCGAAATGGCWGAGAACCA .

-RVic2: GAGTCTTCGAAGTTGTAACC.

GapA :

-Fgap : A173:TGAAATATGACTCCACTCACGG.

-Rgap : A181:CTTCAGAAGCGGCTTTGATGGT.

Mdh :

-Fmdh130: CCCAACTCGCTTCAGGTTCAG

-Rmdh867: CCGTTTTTCCCCAGCAGCAG.

InfB :

-FinfB1: CTCGCTGCTGGACTATATTCG .

-RinfB1: CGCTTTCAGCTCAAGAACTTCET.

TonB :

-FtonB1:CTTTATACCTCGGTACATCAGGTT.
-RtonB2 :ATTCGCCGGCTGRGCRGAGAG.

PgiF :

-Fpgi1:GAGAAAAACCTGCCTGTACTGCTGGC

-Rpgi1: CGCGCCACGCTTTATAGCGGTTAAT.

PhoE :

-FphoE6041: ACCTACCGCAACACCGACTTCTTCG. -RphoE6042:TGATCAGAACTGGTAGGTGA.

PCR conditions

PCR amplification was performed at an hybridization temperature of $50^{\circ} \mathrm{C}$ for all genes except gapA $\left(60^{\circ} \mathrm{C}\right)$ and tonB $\left(45^{\circ} \mathrm{C}\right)$.

PCR cycles

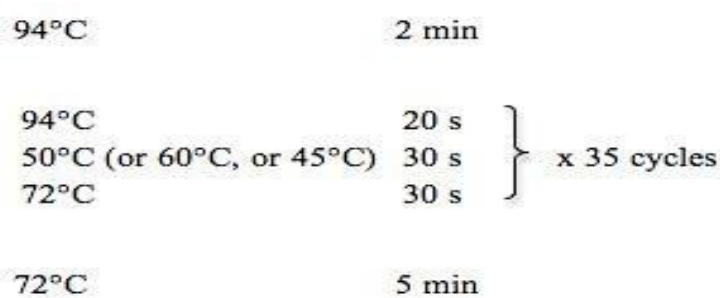


Sequencing of the seven household genes

For sequencing, PCR primers were also used for sequencing, except for the infB gene, for which the infB2F primer was used instead of the forward PCR primer, and for pgi, for which the primers pgi2 $\mathrm{F}$ and pgi2 $\mathrm{R}$ were used.

-infB2F:ACTAAGGTTGCCTCCGGCGAAGC. -pgi2F:CTGCTGGCGCTGATCGGCAT et -pgi2R:TTATAGCGGTTAATCAGGCCGT .

Size of PCR products

-gapA: 662 ;

-infB: 462

-mdh: 756

-pgi: $566-570$

-phoE: 602

-rpoB: 1075

-tonB: 539

Bioinformatics tools

MLST genotyping

For molecular genotyping by MLST technique, we used ML analysis software.

For the genes detected, the comparison took place in the GenBank database (http://www.ncbi.nlm.nih.govgenbank/).

ST: START2 (Sequence Type Analysis and Recombinant Assays 2 ), this database primarily contains allele profile and sequence definition data for MLST patterns, hosted at PubMLST.org.

The site uses the BIGSdb platform (BacterialIsolateGenomeSequenceDatabase). Further details on BIGSdb can be found in Jolley and Maiden (2010), BMC Bioinformatic 11: 595

New sequences were submitted in START 2 software, BIGSdb's BLAST algorithm identified already known sequence variants or flagged an unknown variant for conservative verification. After verification by a conservateur, a new allele number was assigned.

\section{Results and Discussion}

Among 4200 patients, 114 (03\%) had an infected (or colonized) stone, mean age 48 years, male (64\%), mean stone size $15 \mathrm{~mm}$, re- nal site (70\%) with $80 \%$ lumbar pain, $48 \%$ hematuria and $39 \%$ urination burn.

Preoperative ECBU was 90\% negative, cytobacteriological examination of pyelic urine (ECBUP) was $54 \%$ positive including $60 \%$ hematuria and 20\% leukocyte cells on direct examination. The urealytic species found: 22\% Proteus mirabilis, 15\% Staphylococcus aureus, 11\% K. pneumoniae. Emerging species: 12\% Pseudomonas. sp, 09\% E. coli and 08\% Enterobacter cloacae complex (ECC).

Antibiotic resistance: $60 \%$ of $E$. coli and $40 \%$ of $K$. pneumoniae resistant to Cefazolin (CIIG) with $60 \%$ resistance to ciprofloxacin in E. coli and $20 \%$ in K. pneumoniae.

We had 05 extended spectrum $\beta$-lactamase strain: 02 E. cloacae complex and $03 \mathrm{~K}$. pneumoniae with $0 \%$ resistance to carbapenem.

According to table 01, we have summarized the majority of multiresistant bacteria found in urinary stone culture.

By elsewhere. the photo 01 shows an example of klebsiella pneumoniae isolated from urinary calculus and positive with CMI strip E-test * for detection of extended spectrum.

$\beta$-lactamase. The following table summaries the strains of bacteria beta-lactamase with extended spectrum found in culture of urolithiasis.

Picture 2 shows, Extended spectrum $\beta$-lactamase genes detected: CTX-M-15 in K pneumoniae.

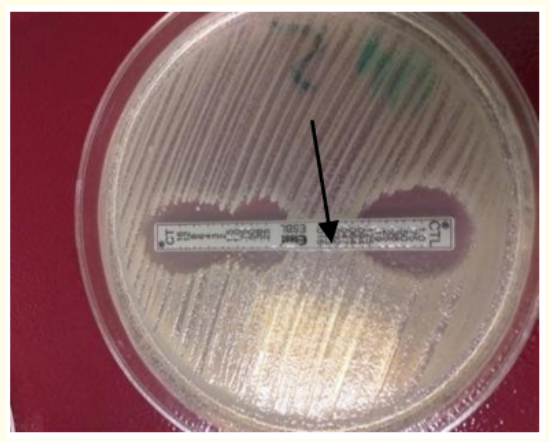

Picture 1: K. pneumoniae (1-3-6) $\beta$-lactamase positive by E- test strip. 


\begin{tabular}{|l|c|c|c|c|c|}
\hline Antibiotics & $\begin{array}{c}K p \\
(1)\end{array}$ & $\begin{array}{c}\text { E. cloacae- } \\
(2)\end{array}$ & $\begin{array}{c}\text { Kp } \\
(3)\end{array}$ & $\begin{array}{c}\text { E. cloacae } \\
(4)\end{array}$ & $\begin{array}{c}K p \\
(6)\end{array}$ \\
\hline AMP/TIC/PIP & $\mathrm{R}$ & $\mathrm{R}$ & $\mathrm{R}$ & $\mathrm{R}$ & $\mathrm{R}$ \\
\hline $\begin{array}{l}\text { Clavulanate } \\
\text { AMC }\end{array}$ & $\mathrm{R}$ & $\mathrm{R}$ & $\mathrm{R}$ & $\mathrm{R}$ & $\mathrm{S}$ \\
\hline Cefazolin & $\mathrm{R}$ & $\mathrm{R}$ & $\mathrm{R}$ & $\mathrm{R}$ & $\mathrm{R}$ \\
\hline Cefoxtin & $\mathrm{S}$ & $\mathrm{R}$ & $\mathrm{S}$ & $\mathrm{R}$ & $\mathrm{S}$ \\
\hline Céfotaxim & $\mathrm{R}$ & $\mathrm{R}$ & $\mathrm{R}$ & $\mathrm{R}$ & $\mathrm{R}$ \\
\hline $\begin{array}{l}\text { ERT. } \\
\text { (CMI<0.2 } \mu \mathrm{g} / \mathrm{Ml})\end{array}$ & $\mathrm{S}$ & $\mathrm{S}$ & $\mathrm{S}$ & $\mathrm{S}$ & $\mathrm{S}$ \\
\hline Acide nalidixic & $\mathrm{R}$ & $\mathrm{R}$ & $\mathrm{R}$ & $\mathrm{R}$ & $\mathrm{R}$ \\
\hline Ciprofloxacin & $\mathrm{S}$ & $\mathrm{R}$ & $\mathrm{R}$ & $\mathrm{R}$ & $\mathrm{S}$ \\
\hline Amikacin & $\mathrm{S}$ & $\mathrm{S}$ & $\mathrm{S}$ & $\mathrm{S}$ & $\mathrm{S}$ \\
\hline Gentamycin & $\mathrm{S}$ & $\mathrm{S}$ & $\mathrm{S}$ & $\mathrm{S}$ & $\mathrm{R}$ \\
\hline Fosomycin & $\mathrm{S}$ & $\mathrm{NT}$ & $\mathrm{S}$ & $\mathrm{S}$ & $\mathrm{NT}$ \\
\hline $\begin{array}{l}\text { Piperacillin-Tazo- } \\
\text { bactem (CMI 50 } \\
<04 \mu \mathrm{g} / \mathrm{mL} \text { ) }\end{array}$ & $\mathrm{S}$ & - & $\mathrm{S}$ & - & $\mathrm{NT}$ \\
\hline $\begin{array}{l}\text { sulfamethoxazole- } \\
\text { trimethoprime } \\
\text { (Batrim) }\end{array}$ & $\mathrm{R}$ & $\mathrm{R}$ & $\mathrm{R}$ & $\mathrm{R}$ & $\mathrm{R}$ \\
\hline Colistin (CMI) & $\mathrm{S}$ & $\mathrm{S}$ & $\mathrm{S}$ & $\mathrm{S}$ & $\mathrm{S}$ \\
\hline
\end{tabular}

Picture 3 shows, qnr-B 1 genes in Enterobacter cloacae complex and K. pneumoniae.

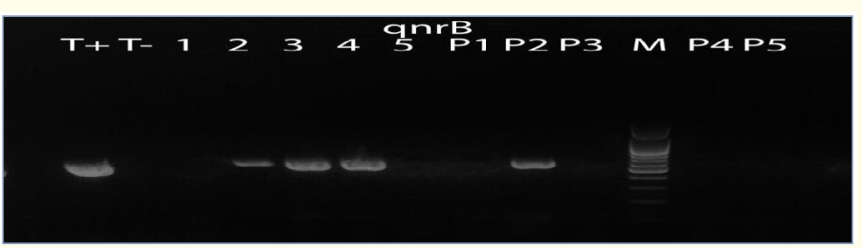

Picture 3: Gel of the products amplified by PCR qnrB1 after electrophoresis on agarose gel (1.5\%).

T+: Klebsiella pneumoniae plasmidp NDM-MAR - qnrB1 +, T-: Distilled Water, P: Plasmid DNA, M: Size marker (100-1000-3000bp).

The Geno typing of K pneumoniae isolated from calculus detected two new and specific clone Sequence type, ST: 29 and 405, as it is to represent in the following table and phylogenic tree in figure 1.

\begin{tabular}{|l|c|c|c|c|c|c|c|c|}
\hline $\begin{array}{l}\text { Bacté- } \\
\text { ries } \\
\text { ESBL+ }\end{array}$ & ST & gapA & infB & Mdh & Pgi & rpoB & phoE & tonB \\
\hline Kp-1 & 405 & 2 & 1 & 2 & 3 & 4 & 10 & 110 \\
\hline kp-3 & 405 & 2 & 1 & 2 & 3 & 4 & 10 & 110 \\
\hline kp-6 & 29 & 2 & 3 & 2 & 2 & 4 & 6 & 4 \\
\hline
\end{tabular}

Table 2: Typical sequences of ESBL positive Klebsiella pneumoniae strains.

Kp: K. pneumoniae beta-lactamase positive from calculus/stone culture. ESBL: Extended spectrum $\beta$-lactamase.

The phylogenetic tree was generated using MEGA 06 computer software (Tamura., et al. 2013) with the maximum likelihood bootstrap algorithm and the TamuraNei model based on concatenate sequences of loci to find the clusters.

According to Logan L.-K. (2019) [2], the enterobacteriaceae resistance pandemic remains one of the significant public health threats of our time.

Uropathogenic Enterobacteriaceae are associated with morbidity and mortality significantly in infected individuals, and a growing number of reports describe the intestinalmicrobiota (flora), and therefore the urinary microbiota, as a probable source of micro-organisms harboring extended spectrum enterobacteriaceae (EBLSE) or carbapenemases (ex, bla KPC-CTXM), contribu- 
ting to the main growing public health problems, in particular infectious complications which may be linked to the transplant of the latterin a urinary stone [3-6].

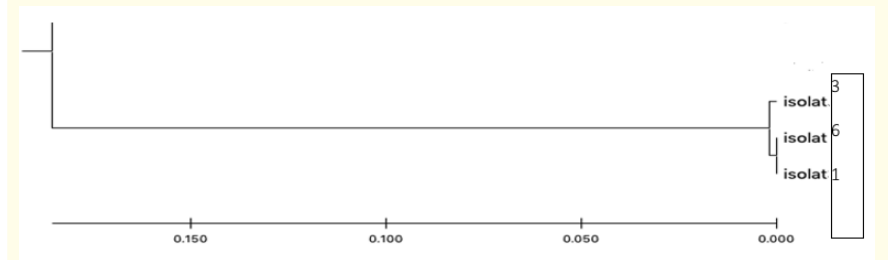

Figure 1: Phylogeny between K. pneumoniae $\beta$-lactamase positive (1-3-6).

Indeed, E-ESBLs are found in a large proportion of Gram-negative bacilli [119.120]. In our phenotypic and genotypic study, bacterial isolates were found in a deep infectious deposit and generated $\beta$-lactamase-producing Enterobacteriaceae extended ESBL spectrum, genetically confirmed and estimated at five strains, i.e. $7.5 \%(5 / 67)$ in an infectious stone.

The three clinical strains (2 Enterobacter cloacae complex and one $K$. pneumoniae) had resistance to fluoroquinolones, but none of them were resistant to carbapenem(ertapenem) or aminoglycosides (amikacin and gentamycin). Subsequently, we described in $K$. pneumoniae the presence of BLSE genes of the bla type CTX-M15 resistant to $\beta$-lactams, which is consistent with the results of their antibiograms (Table 1), in particular with third generation cephalosporins.

This $\beta$-lactam resistance gene has already been described in Algeria and France inprevious studies (Barguigua., et al. 2013; Nedjai., et al. 2012). It has also been described inMexico and Spain (Oteo., et al. 2013) [7-9].

Diffusion of the CTX-M15 gene has been described by the authors as the result of wide dissemination of the same plasmid or related plasmids which have the samegenetic carrier, CTX-M15, first identified in an epidemic clone of $E$. coli is currently the most responded ESBL gene in the world [10].

Our data are also consistent with the results of other authors who suggest that CTX-M producing K. pneumoniae isolates are genetically heterogeneous.
The emergence and polyclonal spread of CTX-M-producing K. pneumoniae has occurred probably produced among isolates of various genetic origins.

In clinical strains, genes encoding CTX-M are usually located on plasmids varying in size from $7 \mathrm{~kb}$ to $160 \mathrm{~kb}[11,12]$.

Transmission of CTX-M genes in Enterobacteriaceae is mediated by plasmids or by several mobile genetic elements already described in the literature. Given the predominance of CTX-M15 genotypes among genetically heterogeneous isolates of K. ESBL pneumoniae, our study also objectified the transfer of genetic elements carriers of blaCTX-M15 via the plasmid (P5) revealed in the gel (Picture 2). Several authors have describes these ESBL gene variants of the CTX-M type which were already characterized by theworld, such as blaCTX-M1 in France (Biendo., et al. 2008) and in Algeria (Nedjai., et al. 2012) [9,14].

Among the predominantly found species, we certainly had a non-significant number of K. pneumoniae belonging to the same ST clone, but we were able to assess globally the clonal link that exists in our clinical strains of Enterobacteriaceae isolated from urinary calculi. Infection. MLST analysis showed that the main types of sequences(ST) identified among the Kp ESBL + 5 (3/5) isolates, in our series, were ST405 $(n=2)$ and a new ST $29(n=1)$ added to the MLST database of K. pneumoniae.

The other two STs identified in our study (ST405) have been described in Europe and South America in strains of human origin. In addition, a clinical strain K. pneumoniae ST405 was detected in Algeria (Oran Military Hospital) with a carbapenemase. However, Machuca., et al. In 2016, published the first report of a K. pneumoniae ST405 without harboring carbapenemase, which is consistent with our strains clinics isolated in the «infectious calculus biofilm» $[15,16]$.

Regarding the result of molecular typing by MLST of the ST29 type of our strain K. pneumoniae (6) ESBL +, we found that it was found in a previous study (Yang and Zhang, 2008), in strains of $k$. pneumoniae studied in Japan.

In France, Laure Surgersa, in 2019, objectified their association in the in vitro formation of infectious biofilm. So, it could be that this virulence factor participates in said infectious stone formation and dissemination of said resistance to antibiotics or measures. 
prevention of their clinical recurrence, recurring public health problems $[17,18]$.

In addition, the strains K. pneumoniae ST29 and ST405 clustered together the tree Phylogenic (figure 1). Which means that the same strain of $K$. pneumoniae circulated in the wards of urology, the operating room and the emergency department of Mohamed Seghir Nekkache Hospital.

Transmission could have been manipulated by staff or by a cystoscope contaminated, used to place double J catheters, especially in medical units urology. The probable port of $k$. pneumoniae ESBL + (and carbapenemase) in the microbiotaintestinal infection is a possibility due to undocumented and untimely treatments taken later in town or in the community, resulting in their silent selection $[8,11,19]$.

\section{Conclusion and Perspective}

The infectious calculus can be a reservoir of virulent or pathogenic microorganisms (bacteria, yeast, anaerobes...) multi-resistant or highly resistant.

We got 7.5 percent, or five ESBL bacteria positive from the urinary stone. Phenotypic explorationand especially molecular germs found in the calculus (or its fragments) allowed usto follow the underhanded evolution of the antibiotic resistance of the CTX-M-15 genes of ß-extended-spectrum lactamase (ESBL) and qnr B-1 fluoroquinolones detected in K. pneumoniae (ST405/ST29) and Enterobacter cloacae complex at Mohamed Seghir Hospital Nekkache.

The genotyping of Klebsiella pneumoniae isolated at Mohamed Seghir Nekkache Hospital from the culture of urinary calculus by MLST demonstrated the distribution of strains in two typical sequences different, including two ST 405 and a new ST 29 phylogenetically very similar.

Moreover, it is alarming that the Klebsiella pneumoniae and Enterobacter cloacae complex are carriers of $\beta$-lactamase at extended spectrum (ESBL +), as well as by the presence of Pseudomonas $s p$ with several virulence and natural resistance factors (slime) to $\beta$-lactams (cephalosporins I, II and 3rd generation), and also Enterococcus spp naturally resistant to third generation cephalosporin, which can cause the vital prognosis of patients at the non-negligable cost of care.
Ultimately, prospective analytical studies and especially control cases would be very interesting in the futur to comparing the infectious agents detecte by sequencing tool but weshould correlating all the results to the clinical and epidemiological context of the patients.

\section{Acknowledgements}

I would like to thank you very much the Professors: Boumedinne, Ghaffour, Yala, Bouzeghoub, Benrabah, Benamrouche, Ziane, Rahal, Tali-Maamar, Zerrouki, Azli and Especially Souid.

\section{Limit of the Study}

Our study has a significant limit in terms of the number of recruitment and the number of detection of colonizing or infecting beta-lactamase bacteria. In addition, the search for Anaerobic bacteria has not been carried out

\section{Bibliography}

1. Daudon M. “Epidemiology of urolithiasis”. Paris (France): Elsevier Masson (2018).

2. Logan LK. "A Multi-Centered Case-Control Study of Factors associated with Klebsiella pneumoniae carbapenemase producing Enterobacteriaceae infections in children and young adult". The Pediatric Infectious Disease Journal 38.5 (2019): 490-495.

3. Noël N and Rieu P. "Urinary lithiasis of infection". EMC-Urology 06-02 (2013): 18-104-C-10.

4. Daudon M and Traxer 0. "Urinary lithiasis". Paris (France): Médecine-Sciences-Lavoisier (2012): 672.

5. Nishant P. "Multidrug Resistant Bacteriuria Before Percutaneou Nephrolithotomy Predicts for Postoperative Infectious Complications". Journal of Endourology 15 (2015).

6. De Miguel-Elízaga I and Martínez-Ruiz A. "Kidney stones in a Mediterranean population from the south of Spain". Clinical Chemistry and Laboratory Medicine 50 (2011): 545-548.

7. Oteo J and Orden B. "CTX-M-15 producing urinary E. coli 025 b-ST131phylogroupe Bé has acquired resistance to Fosfomycin". Journal of Antimicrobial Chemotherapy 64 (2009): 712717. 
8. Barguigua A and El Otmani F. "Prevalence and genotypic analysis of plasmid-mediated $\beta$-lactamases among urinary Klebsiella pneumoniae isolates in Moroccan community". Journal of Antibiotics 66 (2013): 11-16.

9. Nedjai M. "Prevalence and characterization of extended spectrum $\beta$-lactamases in Klebsiella-Enterobacter-Serratia group bacteria, in Algeria". Médecine et Maladies Infectieuses 42.1 (2012): 20-22.

10. Saïdani M. "Emergence of carbapenem-resistant OXA-48 carbapenemase-producing Enterobacteriaceae in Tunisia". Journal of Medical Microbiology 61.12 (2012).

11. Belbel Z. "Evaluation de la résistance aux antibiotiques des souches deKlebsiella pneumoniae isolées dans les hôpitaux d'Annaba". Microbiologieappliquée (2014).

12. Titelman E. "Antimicrobial susceptibility to parenteral and oral agents in a largely polyclonal collection of CTXM14 and CTXM15 producing Escherichia coli and Klebsiella pneumoniae". Journal Pathology of Microbiology and Immunology 119.12 (2011).

13. Bonnet R. "A Novel CTX-M $\beta$-Lactamase (CTX-M-8) in Cefotaxime-Resistant Enterobacteriaceae Isolated in Brazil". American Society of Microbiology 44.7 (2000): 1936-1942.

14. Biendo M. "Successive emergence of extended-spectrum $\beta$-Lactamase-producing and Carbapenemase-producing Enterobacter aerogenes Isolates in a University Hospital". American Society of Microbiology 46.3 (2008).

15. Aggoune N. "Wide spread of OX-48 producing Enterobacteriacae in Algerian hospital à four years study". Journal of Infection in Developing Countries 12.11 (2018).

16. Machuca J. "Caractérisation d'une épidémie due à Klebsiella pneumoniae producteur de CTX-M-15 dépourvu du blaOXA48gene appartenant au clone ST405 dans une unité néonatale du sud de l'Espagne". Journal of Antimicrobial Chemotherapy 71 (2016): 2353.

17. Yan D. "Biofilm-forming Klebsiella pneumoniae strains have greater likelihood of producing extended-spectrum beta-lactamases". Journal of Hospital Infection 68.4 (2004): 369-371.

18. Surgersa L. "Biofilm formation by ESBL-producing strains of Escherichia coli and Klebsiella pneumoniae". International Journal of Medical Microbiology 309 (2019): 13-18.
19. Messai $Y$ and Iabadene H. "Prevalence and characterization of extended-spectrum $\beta$-lactamases in Klebsiella pneumoniae in Algiers hospitals (Algeria)". Pathologie Biologie 56.5 (2008): 319-325.

\section{Assets from publication with us}

- Prompt Acknowledgement after receiving the article

- Thorough Double blinded peer review

- Rapid Publication

- Issue of Publication Certificate

- High visibility of your Published work

Website: www.actascientific.com/

Submit Article: www.actascientific.com/submission.php

Email us: editor@actascientific.com

Contact us: +919182824667 